International Journal on AdHoc Networking Systems (IJANS) Vol. 2, No. 1, January 2012

\title{
Congestion Control and QOS Improvement for AEERG protocol in MANET
}

\author{
S.Rajeswari ${ }^{1}$, Dr.Y.Venkataramani ${ }^{2}$ \\ ${ }^{1}$ Associate professor, Dept. of Electronics and Communication Engineering, Saranathan \\ college of Engineering. \\ rajee_ravi@sify.com \\ ${ }^{2}$ Principal, Saranathan college of Engineering, \\ diracads@saranathan.ac.in.
}

\begin{abstract}
QOS improvement has been a subject of intensive discussion. The research in the network field for congestion control is by means of scheduling packets from different traffic flows for processing at a specific node. When that particular node is selected for the transmission of all traffic flows since it has been chosen as an emerging node for the shortest path in the adaptive energy efficient algorithm, queue scheduling disciplines have been used to improve the quality of service. In this paper, we evaluate the performance of four queuing disciplines (FIFO, PQ, RED and WFQ) which is implemented in the AEERG protocol. This paper gives the NS-2 simulation results to compare their relative performance based on queuing delay, packet drop rate and end-to-end delay for the above queuing techniques.
\end{abstract}

\section{KEYWORDS:}

Ad hoc Networks, Queuing schedule, Drop-Tail, Priority, RED, WFQ.

\section{Introduction}

Ad Hoc network is a wireless, mobile, multi-hop self-organizing network, no infrastructure. The node's mobile features of Ad Hoc network make the network topology change frequently. They are connected through wireless channel, there is no specific routing facilities, each node in the network also acts as a router, forwarding data packets for other nodes, there is also no naming service, directory services and other functions --- these features make the traditional wired networks Quality of Service policies no longer apply to mobile Ad Hoc Networks[1]. The current routing protocols of Ad Hoc networks proposed by IETF are all suitable to a certain network environment, to our knowledge, there is no a protocol itself has QoS mechanism [2]. In a mobile environment, the changing of queue is quite different from those in static conditions.

DOI : $10.5121 /$ ijans.2012.2102 
International Journal on AdHoc Networking Systems (IJANS) Vol. 2, No. 1, January 2012

The cheapest and most common approach to achieve Qos is scheduling mechanisms and packet discarding policies. A queue scheduling discipline manages the allocation of network resources among different traffic flows by selecting the next packet to be processed. Also when packets arrive faster than that they can be processed, arriving packets are dropped and thus controlling the congestion in the network can be influenced. By assigning different weights to various flows in the traffic, proper selection of the packet to be processed can be done. Furthermore, it can reduce the impact of ill-behaved flows on other flows especially when having a mixture of real-time and non-real-time traffic. The queuing disciplines considered in this paper are: First-In-First-Out (FIFO), Priority Queueing (PQ) and WFQ. We also scrutinize the effect of two dropping policies, drop-tail and random-early drop (RED) [7], on their performance.

The remainder of the paper is organized as follows. In the next section, we review related work and briefly discuss the operational aspects of the selected queue scheduling disciplines with some comments on their performance in section 3. We describe the simulation model and traffic scenarios with the simulation results in Section 4. Finally, we conclude our paper and summarize results in Section 5.

\section{RELATED WORK}

Shensheng Tang and Wei Li [3] discussed about an analytical traffic (Markov) model and three queue management schemes are developed for a heterogeneous multihop mobile ad hoc network (MANET). Babak Abbasov[5] proposed a new active queue management algorithm based on RED, called AHRED has been designed and compared with different AQM schemes. Peter Marbach proposed a distributed scheduling and active queue management mechanism for wireless ad hoc networks which is based on a random access scheduler. P. G. Kulkarni et al. presented a proactive prediction based queue management scheme called PAQMAN [7] that captures variations in the underlying traffic accurately and regulates the queue size around the desirable operating point. PAQMAN harnesses the predictability in the underlying traffic by applying the Recursive Least Squares (RLS) algorithm to estimate the average queue length for the next prediction interval given the average queue length information of the past intervals. Jamal N. AI-Karaki et al. propose a QoS routing protocol, called Quality Virtual Routing (QVR), for heterogeneous [8] MANETs. QVR operates on a fixed virtual rectilinear architecture (called virtual grid architecture) that is built on top of the physical topology which consists of a few, but possibly more powerful, mobile nodes known as ClusterHeads (CHs) that are elected periodically and discover multiple QoS routes on the virtual grid using an extended version of the Open Shortest Path First (OSPF) routing protocol and an extended version of WFQ scheduling policy that takes into account the wireless channel state. Liu Ping et al. proposed a mathematical model to calculate the queue delay [10] for MANET, which has a better effect on improving the multimedia stream with delay sensitive. Jianyong Chen et al. a new self-tuning RED [2] is proposed to improve the performance of TCP-RED network.

Hesham N. Elmahdy et al. studied the effect of the packet size and the effect of random early detection (RED) parameters [1] on the Two Rate Three Color Marker (trTCM) and Single Rate Three Color Marker (srTCM). P. Kulkarni et al. presents a predictive queue management strategy named PAQMAN that proactively manages the queue, is simple to implement and requires negligible computational overhead (and hence uses the limited resources efficiently). Xinyu JIN et al. implemented a novel minimum energy routing scheme based on the mobile node's energy 
International Journal on AdHoc Networking Systems (IJANS) Vol. 2, No. 1, January 2012

consumption [9] and the hierarchical node's congestion levels, which is named RED based Minimum Energy Routing (REDMER) scheme. S.Radha Rammohan et al. proposed an effective architecture system [11] to provide a good Quality of Service in Mobile Ad hoc Network.

\section{Proposed queue implementation in AEERG Protocol}

In this protocol, the nodes can be in active mode with probability 1-p or sleep mode with probability $\mathrm{p}$ which is fixed at the initial stage. We set a counter $\mathrm{B}$ to adapt the number of neighbors to which a packet is forwarded. B represents the current number of neighbors at each node which are kept in active state. The value of B is adaptively adjusted based on the packet delivery ratio. This results in less energy consumption[12] and more reliability in the communication networks. When a particular node is selected for the forwarding of packets from different flows, congestion will occur. To ease the congestion and to increase the throughput, different scheduling mechanisms are implemented and the results are discussed.

First-In-First-Out (FIFO) queuing is the most popular queue scheduling discipline that has been widely analyzed in the literature. It also acts as a baseline measure for comparing the performance of other queue scheduling disciplines. In FIFO queuing, all packets are placed in a single queue and then processing will begin in the same order on which they arrived. Hence, the other name is known as first come-first-serve (FCFS) queuing. Although it is simple to implement and has predictable performance in behavior and comparatively low computational load on the system, it has some serious drawbacks for wireless network traffic. It is not capable for providing differentiated service and can not identify to isolate the effect of ill-behaved flow with other flows. A heavy flow can consume the entire buffer space and causes all other flows to be denied service until after the burst is serviced. This can result in increased delay, jitter and loss for the other well-behaved flows traversing in the queue. To reduce the impact of ill-behaved sources, other queue disciplines have been proposed to isolate traffic flows into separate queues. These queues can be serviced according to some scheduling scheme. Among these are priority queuing, fair queuing, and weighted fair queuing, weighted round robin or class-based queuing, and deficit weighted round robin.

Priority Queuing (PQ) is a simple mechanism to provide differentiated services to different packet flows. Packets of different flows are assigned a priority level according to their QoS requirements. When packets reach at the link output, they are first separated into different classifications, enqueued separately in different flows based on their priorities. Then, queues are served in order. The highest priority queue is served first before serving lower priority queues. Packets in the same priority class are serviced in a FIFO manner. As soon as highest-priority packets are served, packets from the lower priority class are served. But if a higher-priority packet arrives while serving a lower-priority packet, the server waits till to complete the service of the current packet then goes back to serve the higher priority queue (non-preemptive PQ). The limitation of PQ is that lower-priority flow packets may receive little attention when a higherpriority class has a continuous stream of packets. This problem is known as starvation problem [1]. Also it does not show fairness to all queues. The closed form analytical solution of a twoclass priority queue is given in [8]. 
International Journal on AdHoc Networking Systems (IJANS) Vol. 2, No. 1, January 2012

Random early detection (RED): It is also known as random early discard or random early drop and it is also an active queue management algorithm. It is used for a congestion avoidance algorithm. This algorithm plays an important role by the way of not admitting full queues for processing, reducing the packet delay and loss. It monitors the average queue size and average number of dropped packets based on statistical probabilities. The RED is also known as a threshold based queuing discipline. It statistically starts of dropping the packets from flows before it reaches its threshold value. So it is considered a good queue for situations where the complexity of per-session state tracking needed by fairness queuing is not affordable. Assume that each node has a single buffer.

Let $\lambda \mathrm{n}$ be the packet arrival rate to the buffer at node $\mathrm{n}$;

Let $D_{n}$ be the expected delay of a packet at node $n$;

Let $\mathrm{P}_{\mathrm{n}}$ be the probability that a packet is dropped at node $\mathrm{n}$ due to a buffer overflow.

For the case of $\lambda=N$,

$$
X(\lambda)=\sum_{1}^{N} \lambda n
$$

Let $\mathrm{X}(\lambda)$ be the network throughput under the network arrival rate $\lambda$. We list the schedulers with the following property.

Property: For a single-cell wireless network consisting of nodes $\mathrm{n}=1, \ldots, \mathrm{N}$, we assume that a scheduler implements a distributed buffer with service rate if the following conditions are true.

(a) The expected delay $D_{n}$ is identical at all nodes, i.e. we have $D_{n}=D$, for values $n=1, \ldots, N$.

(b) The packet-drop probability $\mathrm{P}_{\mathrm{n}}$ is identical at all nodes, i.e.

we have $P_{n}=P$, for values $n=1, \ldots, N$.

(c) The throughput $X(\lambda)$ is a non-decreasing function in $\lambda$ with

$\operatorname{Lim} X(\lambda)=$.

$\lambda \rightarrow \infty$

The above property states that a fair scheduler should serve packets as if the network traffic shares a common buffer that is served at rate, i.e. all packets entering the network should experience the same expected delay and the same probability of being dropped.

For every packet arrival, the RED gateway calculates the weighted moving average queue size (avg). It then compares the results with minimum $\left(\min _{\mathrm{th}}\right)$ and a following schema $[5,6]$.

- When avg $<\min _{\text {th }}$ the packet is dropped with probability one.

- When $\min _{\text {th }} \leq \mathrm{avg}<\max _{\text {th }}$ the packet is dropped with some probability.

- When $\max _{\text {th }} \leq$ avg the packet is not dropped.

The value of the average queue size (avg) is computed as follows:

After each idle period the average queue size (avg) is additively decreased by a constant $\alpha>0$ and after each busy period the average queue size (avg) is additively increased by a constant $\beta>0$. Note that this rule follows the intuition that average queue size (avg) should be increased when the channel is busy, and be decreased when the channel is idle.

It is well known that by employing FIFO, throughput is not as good as priority Queue which is lesser than RED.

Weighted Fair Queuing (WFQ) :The idea of the Weighted Fair Queuing (WFQ) algorithm can be easily explained as a combination of PQ and FQ algorithms. As in the FQ method, all queues are served so that there is no bandwidth starvation, but some queues have more weight in a sense 
International Journal on AdHoc Networking Systems (IJANS) Vol. 2, No. 1, January 2012

that they receive more service. In other words, a weight is given to each queue to assign different priorities to the queues. Packets are stored into the appropriate queue according to their classification.

WFQ is an approximation of the Generalized Processor Sharing (GPS) system. The algorithm is explained as follows. A finish time is assigned to each packet taking into account the link bandwidth, the number of queues, the weight of queues and the packets length. Afterwards, the scheduler serves the queue where the packet with the minimum finish time is stored. The finish time is used to build the order in which the packet will be transmitted through the link.

WFQ is a complex algorithm requiring per service class state and iterative checking of all states for each packet arrival and departure. Consequently, the WFQ presents scalability problems and cannot be used in environments with high volume of traffic requiring many classes of service. One possible application of WFQ is where the number of service classes is limited to a small number, so that the computational load is limited.

\section{PERFORMANCE EVALUATION}

For queuing disciplines specification, we set the maximum queue size to be 500 packets. There are three types of classes in all queues (except FIFO) whose queue buffer size increases from highest priority to lowest one. For enabling RED, we used minimum and maximum threshold as 100 and 200 respectively while keeping the mark probability denominator (the fraction of packets dropped when the average queue size is at maximum threshold) as 10 . We set exponential weight factor (used to calculate average queue size based on the previous average and current queue size) to be 9. Finally for WFQ the different finish time of packets such as 30,75 and 135 and also varying bandwidths such as $1,1.25,1.5$ and 2 Mbps are allocated for flows.

\subsection{SIMULATION PARAMETERS}

NS2 is used to simulate the proposed algorithm. In our simulation, the channel capacity of mobile hosts is set to the same value: 2 Mbps. For the MAC layer protocol the distributed coordination function (DCF) of IEEE 802.11 (for wireless LANs) is used. It has the functionality to notify the network layer about link breakage.

In the simulation, mobile nodes move in a 600 meter $\mathrm{x} 400$ meter region for 50 seconds simulation time. The number of mobile nodes is varied from 10-60. We assume each node moves independently with the same average speed. All nodes have the same transmission range of 250 meters. In our simulation, the speed is set as $20 \mathrm{~m} / \mathrm{s}$. The simulated traffic is Constant Bit Rate (CBR). The pause time of the mobile node is kept as 20-120 sec.

\subsection{PERFORMANCE METRICS}

The simulated values of Throughput and Packet delivery ratio with the increasing number of nodes, Different size of packet and for varying Simulation Pause Time are plotted as shown in the figures below. 
International Journal on AdHoc Networking Systems (IJANS) Vol. 2, No. 1, January 2012

\subsubsection{Throughput:}

The successful number of packets received by the destination is termed by Throughput and it is measured by bytes/sec. For varying number of nodes, Throughput is measured for different queuing and the figure is plotted as in Fig.1.

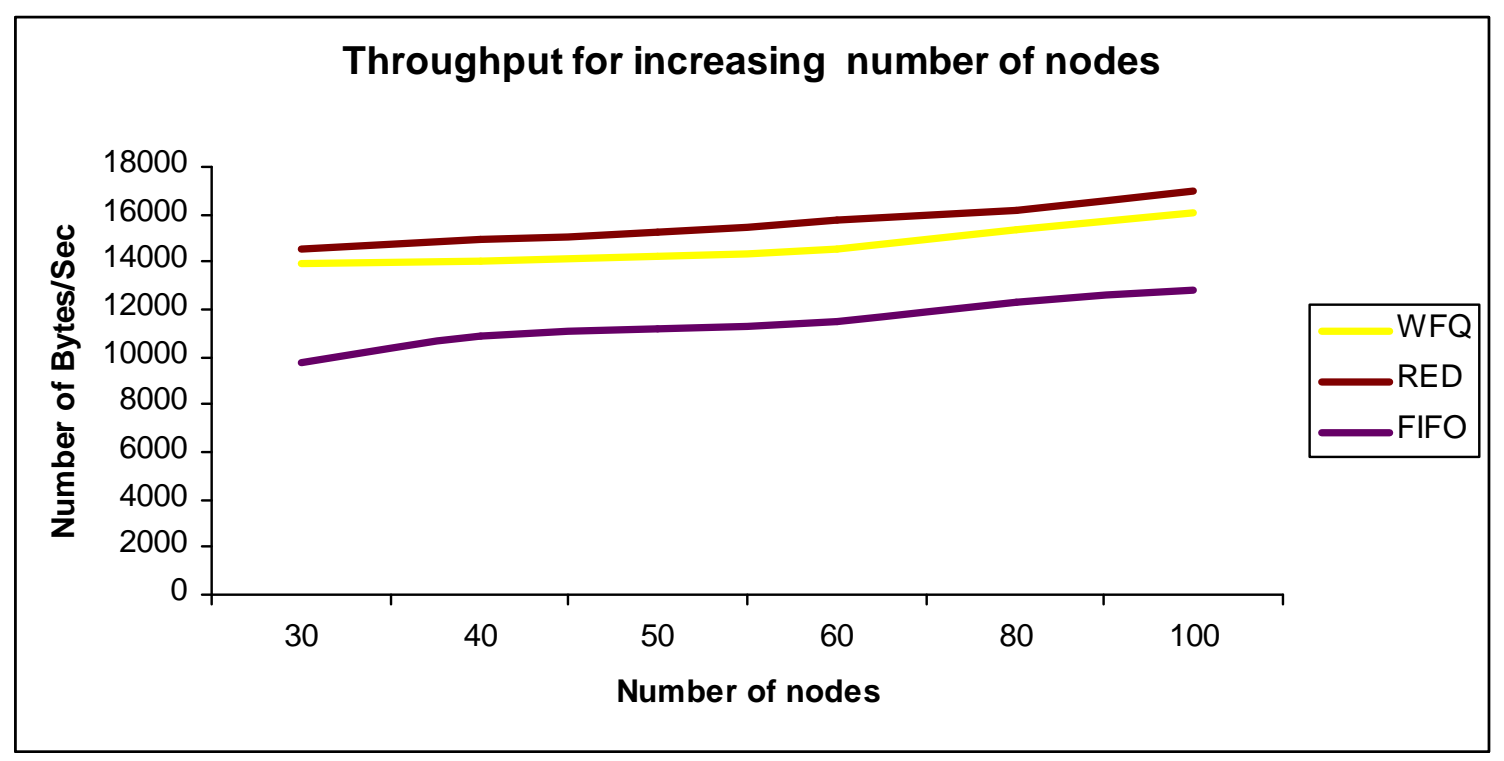

Fig.1 Throughput Vs Number of Nodes

Fig.1 gives the Throughput of three queue scheduling mechanisms when the number of nodes is increased. When the number of nodes is increased, number of flows will increase leads to many number of packets. From the figure, we can see that if we employ FIFO, more number of packets will drop due to fixed buffer size. By means of WFQ more throughput can be achieved.

\subsubsection{Packet Delivery Ratio:}

Packet delivery ratio is calculated by dividing the number of packets received by the destination through the number of packets originated by the application layer of the source. It specifies the packet loss rate, which limits the maximum throughput of the network. The better the delivery ratio, the more complete and correct is the routing protocol. For different queuing disciplines, Packet Delivery Ratio is computed with increasing number of nodes and the graph is plotted in figure. 2 


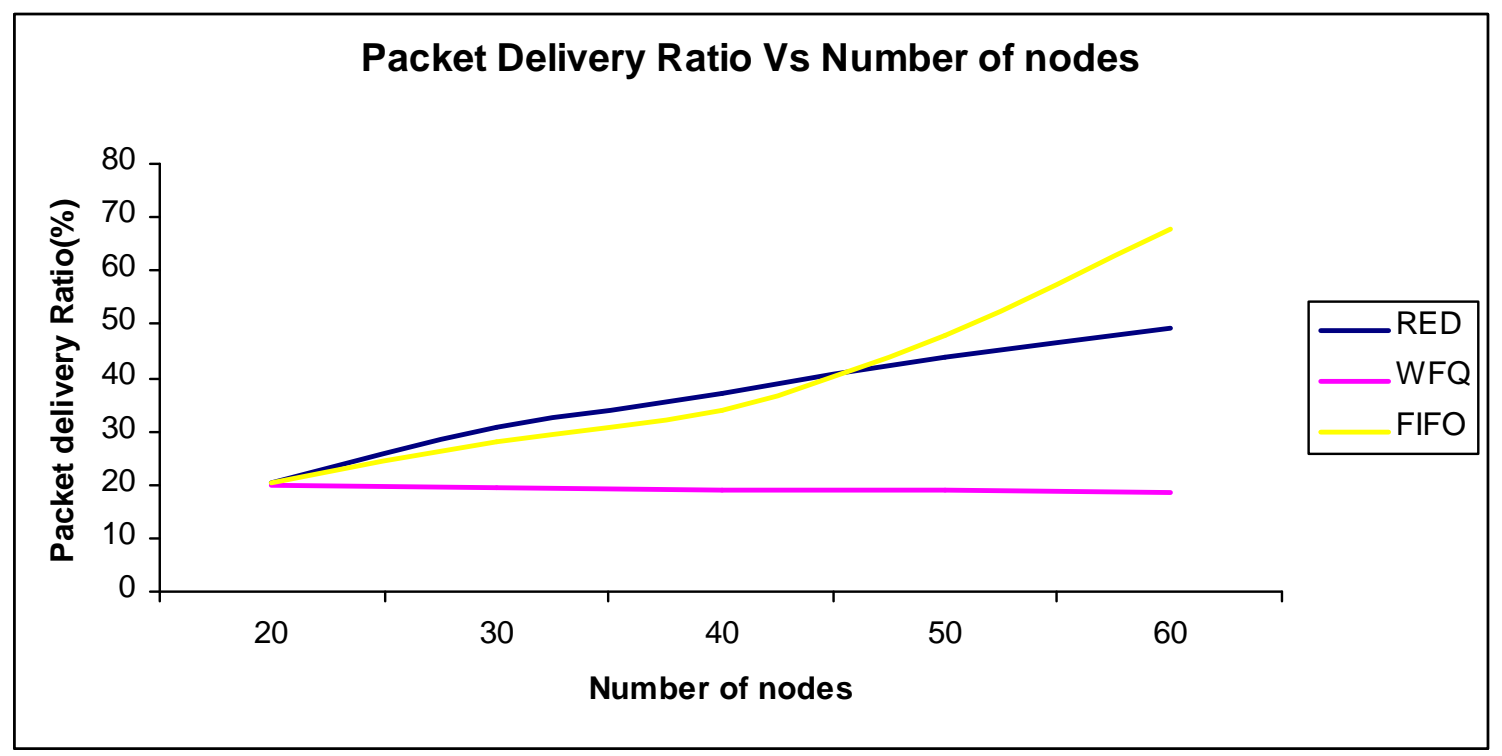

Fig.2 Packet Delivery Ratio Vs Number of Nodes

Fig.3 gives the Packet Delivery Ratio of different queue scheduling mechanisms when the number of packet data is increased. This figure helps us to study about the efficiency of the queue scheduling mechanisms. This result is obtained for non-real time communications. When the number of nodes is increased, number of flows will increase leads to generation of many number of packets.

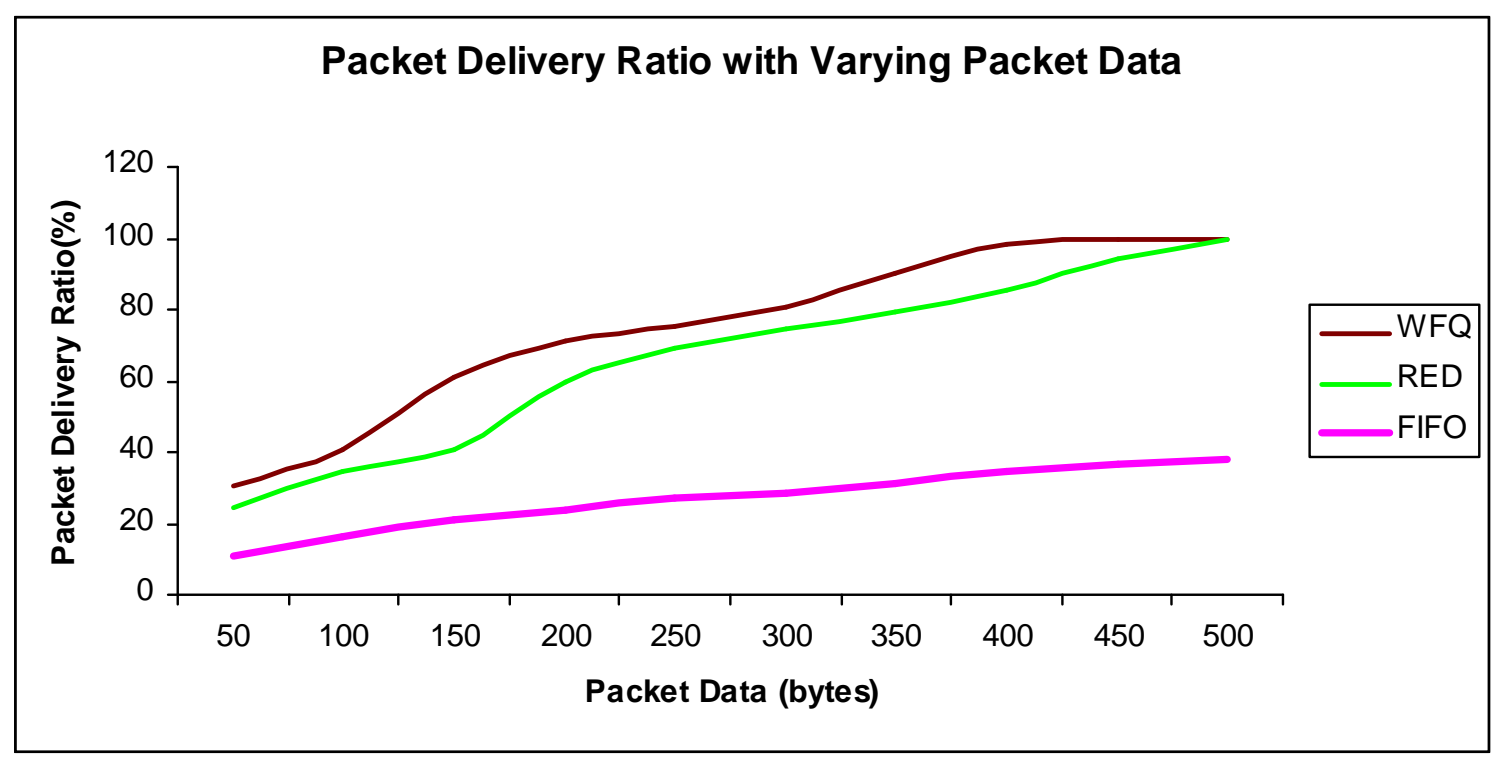

Fig.3 Packet Delivery Ratio Vs Varying Packet Data (bytes) 


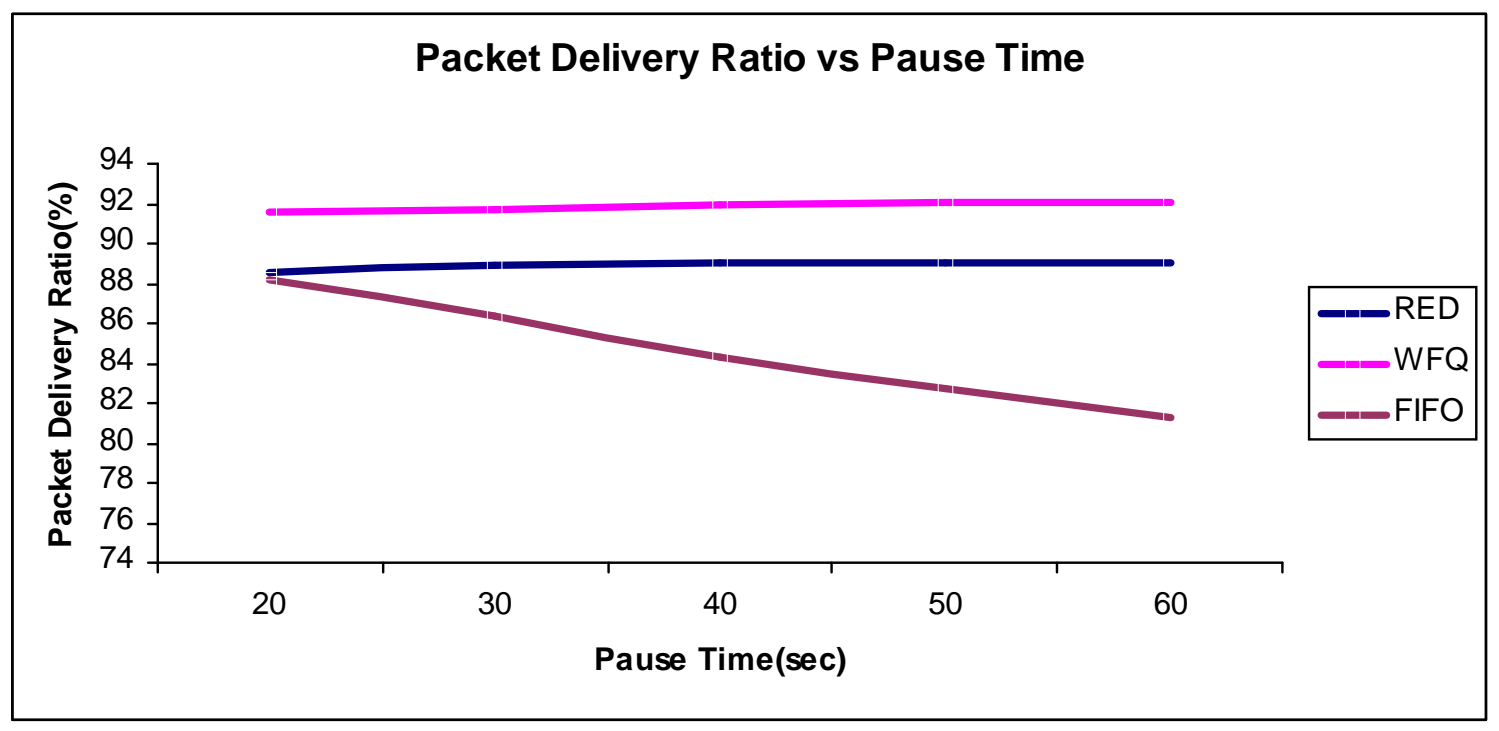

Fig.4 Packet Delivery Ratio Vs Simulation Pause Time

Fig.4 gives the Packet Delivery Ratio of different queue scheduling mechanisms when the pause time is increased. This figure helps us to study about the adaptive queue buffer size change mechanisms. When the Simulation Pause Time is increased, number of free space in the buffer is adaptively changed for RED. But WFQ gives more packets Delivery Ratio than any other queue.

\section{CONCLUSION}

In this study we presented a simulation-based performance evaluation and comparison of three queuing techniques for different number of nodes, packet size and pause time for the impact of using random-early drop as compared to drop-tail policy and weighted fair scheduling. The simulation results show WFQ that outperforms other disciplines in terms of Throughput and packet delivery ratio although RED are also very close to it for the considered node scenarios. We also noticed that using RED has greatly improved all the performance measures especially with FIFO. The reason is that RED monitors the average queue size and randomly drops packets when congestion is detected. Further work is still required to change the type of traffic to examine these queuing disciplines and to study the impact of different traffic scenarios and the use of traffic shapers at the edges of the network.

\section{REFERENCES:}

1. Hesham N. Elmahdy and Mohamed H. N. Taha, "The Impact of Packet Size and Packet Dropping Probability on Bit Loss of VoIP Networks" in ICGST-CNIR Journal, Volume 8, Issue 2, pp. 25-29, January 2009.

2. Jianyong Chen, Cunying $\mathrm{Hu}$, and Zhen Ji, "Self-Tuning Random Early Detection Algorithm to Improve Performance of Network Transmission" in Mathematical Problems in Engineering journal Volume 2011, Article ID 872347, 17 pages , doi:10.1155/2011/872347.

3. Shensheng Tang and Wei Li , "QoS Provisioning and Queue Management in Mobile Ad hoc Networks" in Wireless Communications and Networking Conference, pp.400-405, April 2006. 
International Journal on AdHoc Networking Systems (IJANS) Vol. 2, No. 1, January 2012

4. Shujie Wei, Guangwei Bai , Hang Shen, “An Adaptive Queue Management Mechanism for Video Streaming over Mobile Ad Hoc Networks" in WiCOM'09 Proceedings of the 5th International Conference on Wireless communications, networking and mobile computing, October 2009.

5. Babak Abbasov, "AHRED: A Robust AQM algorithm for wireless Adhoc Networks" in International Conference on Application of Information and Communication Technologies, PP.1-4, October 2009.

6. Peter Marbach, "Distributed Scheduling and Active Queue Management in Wireless Networks" in INFOCOM 2007 :2321-2325.

7. P. G. Kulkarni, S. I. McClean, G. P. Parr, M. M. Black, "Proactive Predictive Queue Management for improved QoS in IP Networks" in Proceedings of the International Conference on Networking, International Conference on Systems and International Conference on Mobile Communications and Learning Technologies, April 2006.

8. Jamal N. AI-Karaki, Ahmed E. Kamal, "Supporting Quality of Service In Mobile Ad hoc Networks" in ACS/IEEE 2005 International Conference on Computer Systems and Applications (AICCSA'05).

9. Liu Ping, Yuan Peiyan, “An Approach to Calculate Queue Delay in Mobile AdHoc Networks" in International Conference of Information Science and Management Engineering, August 2010.

10. Wenyu Cai, Xinyu Jin, Yu Zhang, Kangsheng Chen and Rui Wang, "ACO Based QoS

Routing Algorithm for Wireless Sensor Networks" in Ubiquitous Intelligence and Computing, LNCS 2006.

11. S.Radha Rammohan and K.Rengarajan, "Study of Possible Packets Travelling Algorithm for Effective Mobile Ad hoc Networks" in International Journal of Soft Computing, Volume.4, pp. 162-167, 2009.

12. S.Rajeswari ,Dr.Y.Venkataramani, “An Adaptive Energy Efficient and Reliable Gossip Routing Protocol For Mobile Adhoc Networks” IJCTE, Volume-2, No.5, Oct 2010. 